\title{
Sociología y Ciencias Sociales en tiempos de crisis pandémica
}

\section{Sociology and the Social Sciences in a Time of Pandemic Crisis}

\section{Manuel Fernández Esquinas ${ }^{1}$}

\section{Resumen}

En este artículo se realiza una discusión sobre el papel de la sociología, junto a otras ciencias sociales, para analizar algunos aspectos sociales de la crisis provocada por la pandemia del COVID-19. Para ello se acude a condicionantes sociales provenientes de la estructura social, la cultura, el poder y la autoridad de los científicos y la construcción de instituciones relacionadas con la salud y la I+D. Los efectos de la pandemia se abordan teniendo en cuenta las consecuencias previstas y no previstas del confinamiento. En las conclusiones se incluyen reflexiones sobre el impacto de la crisis en las ciencias sociales.

\section{Palabras clave}

Pandemia, crisis, salud, ciencia, sociología, ciencias sociales.

\section{Abstract}

This article provides a discussion on the role of sociology and other social sciences in order to analyze major social issues of the COVID-19 pandemic crises. Social conditions related to social structure, culture, the power of science and the construction of institutions related to health and R\&D are discussed. Some impacts of the pandemic crisis are considered from the angle of the untended and unintended consequences of confinement. In the conclusions some thoughts about the effects of the crisis on the social sciences are provided.

\section{Keywords}

Pandemic, crisis, health, science, sociology, social sciences.

\section{Cómo citar/Citation}

Fernández Esquinas, Manuel (2020). Sociología y Ciencias Sociales en tiempos de crisis pandémica. Revista de Sociología de la Educación-RASE, 13 (2) Especial, COVID-19, 105-113. http://dx.doi.org/10.7203/RASE.13.2.17113. 


\section{Introducción}

Europa y otras partes del mundo se están enfrentando en 2020 a una de las crisis más importantes de esta generación. El impacto social de la pandemia del COVID-19, junto a las decisiones que se tomen para gestionarlo, puede afectar seriamente a la sociedad en la que vivimos. Frente a estas amenazas muchos colectivos de las ciencias sociales se ven en la obligación de ayudar en la medida de sus posibilidades. En pocas semanas han surgido encuestas, diagnósticos, reportajes fundamentados en evidencias, blogs que recopilan recursos e infinidad de ensayos dirigidos a proporcionar sentido sobre la crisis pandémica o a llamar la atención sobre sus efectos. Aunque los resultados son desiguales y aún es pronto para tener evidencias sobre muchos aspectos de esta crisis, la cantidad y variedad de aportaciones reflejan la vocación de servicio de los profesionales de las ciencias sociales.

La RASE se ha sumado a este esfuerzo de una manera decidida con un número especial dedicado a contribuir al análisis de un fenómeno inédito en su magnitud e impacto. Con esta iniciativa la revista muestra una vez más su compromiso para ponerse al servicio de los problemas sociales más candentes desde la sociología de la educación. Este artículo pretende responder a los objetivos del monográfico con una discusión en torno la pregunta que parece estar en el trasfondo de muchas iniciativas como las mencionadas: ¿qué pueden hacer las ciencias sociales? Y, en particular, ¿qué podemos hacer los sociólogos? El rol de la sociología a veces es difícil de percibir por parte de políticos, colegas de otros grupos profesionales y sociedad civil en general, más allá de algunas herramientas de uso común y de resultados que tienen impacto mediático. Es conveniente intentar hacer más visible nuestro papel frente problemas de gran trascendencia como los actuales. Además, discusiones como las que propone la RASE pueden ser útiles a la comunidad profesional a la hora de reflexionar sobre nuestras estrategias y habilidades.

Intentar explicar el papel de la sociología en unas cuantas páginas entraña bastantes riesgos debido a lo que se debe dejar fuera. En este texto se presenta un argumento sintético, necesariamente incompleto y provisional, sobre la utilidad de la sociología para entender la crisis pandémica en su complejidad y para prever sus efectos. En resumen, en primer lugar puede contribuir ofreciendo conocimientos que permitan entender cómo los fenómenos pandémicos están interrelacionados estrechamente con aspectos sociales. En segundo lugar, puede ofrecer algunas herramientas para orientar la toma de decisiones, documentando de manera rigurosa los impactos sociales, e identificando muchas consecuencias no previstas de la cadena de acciones que la crisis está provocando.

Este argumento se desarrolla de la siguiente manera: el punto dos realiza una síntesis de los principios esenciales de la sociología que pueden aplicarse a la crisis pandémica. El punto tres se ocupa de los factores que funcionan como condicionantes sociales de la pandemia. El punto cuatro se ocupa de algunas consecuencias de la crisis. En las conclusiones se incluyen razonamientos sobre el impacto que la crisis puede tener en las propias ciencias sociales.

\section{Sobre la perspectiva sociológica y su aplicación a la crisis}

Las ciencias sociales no se diferencian mucho del resto de ciencias en lo referido a su vocación práctica: sirven para orientar la toma de decisiones. Aunque muchos de los conocimientos son resultado de la curiosidad humana sobre la vida social, sobre todo se espera obtener criterios rigurosos que ayuden a actuar. Una muestra de ello es que los gobiernos y las empresas constantemente intentan utilizar el conocimiento disponible en las ciencias sociales para llevar a cabo sus propósitos. 
Sin embargo, las ciencias sociales tienen una particularidad que va más allá del uso puramente instrumental: proporcionan una base de conocimiento desde la que las personas entienden el mundo en sociedad. Dicho de manera simple: los virus no leen lo que los científicos publican sobre ellos. Las personas sí tienen por costumbre leer y aprender, aunque sea selectivamente, lo que los científicos sociales publican sobre la vida en sociedad y sobre las interacciones entre naturaleza y sociedad, como es el caso que nos ocupa. Un destinatario del conocimiento social es la propia sociedad civil. A través de la divulgación y de la asimilación de lo que producen las ciencias sociales (en el sistema educativo, en los medios de comunicación, en su trascendencia en las actividades culturales, etc.) las personas adquieren visiones del mundo y criterios de actuación sobre él. Y de esa manera, influyen en las políticas públicas y en los asuntos colectivos. Las ciencias sociales tienen por todo ello un papel muy importante en el análisis de las manifestaciones que adquiere la crisis pandémica y en las formas de actuación colectiva que se pueden adoptar para mitigar sus efectos.

Las distintas ciencias sociales también se parecen bastante entre sí en este propósito general. Sin embargo, ocurre que el comportamiento de las personas en sociedad es algo extremadamente complejo (seguramente mucho más complejo que el comportamiento de cualquier virus). Para entender y explicar unas partes de la realidad se ven en la obligación de acudir a modelos algo más simplificados que abstraen otras partes que no se podrían abarcar conjuntamente. Casi todas las ciencias sociales parten de este tipo de modelos. Y como no se puede reducir la realidad social a las bases cognitivas de una sola disciplina, los esfuerzos más efectivos para comprender fenómenos complejos son multidisciplinares. Se asume que es posible avanzar hacia un mejor entendimiento de la complejidad reconociendo las ventajas y límites de las visiones que aporta cada rama de las ciencias sociales.

El modelo de la sociología tiene una serie de particularidades cognitivas que lo diferencian de los modelos de la psicología y de la economía, por poner solo dos ejemplos, a la hora de observar algunas partes del comportamiento y algunos fenómenos sociales. Se basa en unos cuantos principios axiomáticos que no son ni mejores ni peores. Son puntos de partida que facilitan prestar más atención a aspectos que de otra manera pasarían desapercibidos ${ }^{2}$. Aunque sea de manera muy sintética, en la sociología se pueden citar algunos principios comunes más allá de la gran diversidad de enfoques y teorías. Son los siguientes:

1) Los hechos sociales adquieren cierta autonomía respecto a sus manifestaciones individuales. $\mathrm{O}$ dicho de otra forma, la agregación de conductas individuales termina provocando realidades de tipo colectivo que acaban imponiéndose y afectando a la vida de la gente. 2) Los valores y las normas tienen un papel importante en la orientación de la acción humana. La racionalidad y las emociones también lo tienen, aunque en uno y otro caso suelen estar enmarcadas y moldeadas por las bases culturales de partida. 3) El poder y la influencia, distribuidos desigualmente entre personas y colectivos sociales, tienen una fuerza importante para restringir algunos comportamientos o impulsar otros. 4) Las instituciones se construyen socialmente, o lo que es lo mismo, cada institución que observamos, desde la familia a las grandes corporaciones, depende de procesos sociales concretos. 5) Las iniciativas personales y colectivas siempre se insertan en un contexto de relaciones sociales. El resultado de cualquier estrategia (desde una política hasta el diseño de una institución), depende del entramado de relaciones compuesto por deseos, expectativas y vínculos entre las personas.

2 Se sigue el argumento de A. Portes (2013). Considera estos principios como «conceptos meta-teóricos», que están antes de las teorías, y que por tanto no son verificables empíricamente. Son los puntos de partida que todas las disciplinas se ven en la obligación de utilizar. A partir de ellos se construyen mecanismos explicativos o teorías más conectadas con realidades concretas, y que tienen posibilidades de ser verificados. Aquí se han seleccionado a propósito los principales, haciendo abstracción de otros empleados por algunas teorías sociológicas. 
Más allá de estos principios axiomáticos, cualquier aseveración sobre la sociedad está sujeta a investigaciones que es necesario corroborar. Estos principios se pueden aplicar como punto de partida a multitud de fenómenos. También sirven para resumir cómo la sociología piensa sobre las epidemias ${ }^{3}$.

Las epidemias son fenómenos biológicos, pero también sociales. Epidemiólogos y sociólogos tienen en común la correspondencia a la hora de entender empíricamente los elementos que intervienen en la transmisión. La manera en que se manifiestan las epidemias y se convierten en pandemias depende de la manera en que la gente vive y piensa. La extracción de recursos de la naturaleza, la manipulación de animales, los movimientos de población, las creencias sobre la vida natural, junto a muchas formas de relación social, influencian la interacción con otros organismos biológicos. Ello crea las pautas de salud y enfermedad que todos experimentamos colectivamente. En ocasiones se generan condiciones que nos hacen vulnerables a las infecciones de otros organismos y que provocan su extensión a otras personas. En las siguientes páginas se discuten algunos factores sociales que afectan a la propagación de la actual pandemia acudiendo a los principios indicados antes, siempre a modo de hipótesis sujetas a verificación.

\section{Las bases sociales de la pandemia}

Un primer grupo de factores que afecta a la pandemia tiene que ver con condiciones de la estructura social. El escenario macro de fondo es desde luego el mundo globalizado. La intervención en los ecosistemas, la crianza intensiva de animales para la alimentación y la globalización del comercio y el turismo provocan situaciones que son estructuralmente distintas a las que dieron lugar a otras pandemias de origen animal a lo largo de la historia de la humanidad. Se sabe que ni la transmisión ni los efectos de los virus afectan por igual a todos los países y a todas las clases sociales. La principal brecha ocurre entre países ricos y pobres. Las diferencias en sistemas de higiene básicos, nutrición, hacinamiento, educación e infraestructura sanitaria influyen (Weitz, 2016).

Sin embargo, si bien en esta ocasión la pandemia surge en una región de China, de momento está afectando más en términos relativos a países del primer mundo, y dentro de estos, se está concentrando más en algunos. Esta pandemia ya no es un problema de países pobres debido a la organización de la economía y de los movimientos de población. También influye la estructura de oportunidades desde la que actúan los gobiernos y los sistemas de salud. Si nos centramos en el Sur de Europa, vemos que existen grandes diferencias entre la mayor propagación en Italia y España, los primeros en importar infecciones, y lo ocurrido en Grecia y Portugal. A simple vista se podría pensar, por ejemplo, que Grecia es un país más vulnerable debido a la presión de refugiados provenientes de Oriente Medio y a los mayores recortes que ha sufrido su sistema sanitario. Razones similares se podrían aducir respecto a diferencias con otros países del entorno europeo o del norte de África. ¿Por qué entonces Italia y España están sufriendo más?

Una primera razón posiblemente haya que buscarla en las condiciones provocadas por los movimientos de la población. España recibe entre 80 y 90 millones de turistas extranjeros y en torno a 125 millones de visitas cada año. En este aspecto es uno de los países más interconectados del mundo. Italia también es una potencia turística. Además, los movimientos de población son estacionales en cada zona del mundo. En invierno hay más movimientos desde muchos países hacia España e Italia que hacia otros del norte o del este de Europa. Por ello las probabilidades de transmisión son distintas. Una segunda razón tiene que

\footnotetext{
3 Las explicaciones sobre cómo las condiciones sociales intervienen en las pautas de salud y enfermedad de los individuos, poblaciones y grupos sociales se pueden encontrar en cualquier manual de sociología de la salud. Por ejemplo, Weitz (2016). Un análisis sistemático sobre los determinantes sociales de la salud puede verse en Navarro (2009).
} 
ver con la secuencia temporal de la transmisión entre países y con el tiempo de reacción. El hecho de que la epidemia salte primero a un país en concreto en vez de a otro tiene un componente de aleatoriedad, dentro de las distintas condiciones que crean los movimientos de población. Pero cuando las diferencias a la hora de reaccionar son una cuestión de días, el orden importa. El primer país donde se detecta el contagio tarda en reaccionar más que el segundo o el tercero debido al efecto de aprendizaje. El estado de confinamiento en Grecia se decretó cuando aún había pocos afectados y se sabía un poco mejor lo que había ocurrido en otros lugares. Aunque con pocos días de margen, posiblemente ese fue un factor diferencial. Si hubiesen sido los primeros en importar contagios, los efectos para ese país podrían haber sido mucho peores. Se trata solo de una hipótesis. En cualquier caso, es necesario prestar atención a las situaciones estructurales que están más allá del margen de actuación de gobiernos y ciudadanos. Las ciencias sociales pueden contribuir aportando evidencias para evitar los argumentos simples que concentran culpabilidades en países.

Un segundo grupo de factores condicionantes se refiere los valores que orientan la acción y que se reflejan en formas de vida y relación social. Algunos valores actúan en capas profundas, como las ideas religiosas y las cosmovisiones que afectan a las normas de comportamiento. Los antropólogos que han trabajado en África muestran cómo las normas moldean las epidemias y las medidas de salud pública se tambalean a menudo por razones sociales y culturales. En concreto, durante la crisis del Ébola se demostró la efectividad de cambiar rituales de entierros y ceremonias convenciendo a líderes religiosos legitimados y respetando las costumbres locales, en lugar de acudir a prohibiciones drásticas (Leach et al., 2010). Otros valores se encuentran en capas más superficiales en forma de actitudes positivas o negativas hacia algunas conductas. La presencia de valores comunitarios, que favorecen la responsabilidad grupal y toleran mejor el control del Estado para promover un bien público, pueden marcar una diferencia respecto a otros más individualistas que dan más importancia a la privacidad. Esto puede explicar la disciplina social para controlar la trasmisión lograda por algunos países asiáticos como Corea.

Pero a pesar de la importancia de la cultura, los científicos sociales deben ser extremadamente cuidadosos al atribuir un «factor cultural» a la expansión de una enfermedad aduciendo solamente a los hábitos enraizados en una cultura concreta. En primer lugar, porque las explicaciones basadas en diferencias culturales puede que funcionen empíricamente a gran escala cuando se comparan grandes zonas del mundo, pero dejan de funcionar en estructuras sociales de países o regiones gracias a los procesos de aculturación. En segundo lugar, porque en multitud de lugares las barreras para que se cumplan efectivamente las medidas preventivas se deben a diferencias en los recursos económicos y a desigualdades de clase. Por ello, se necesita de una indagación detallada que evite estigmatizar a determinados grupos sociales.

Un tercer grupo de factores condicionantes tiene que ver con las distintas formas de poder e influencia para actuar frente la pandemia. Las más patentes están relacionadas con el control de recursos económicos y su distribución a través de bienes y servicios, tanto sanitarios como de otro tipo. Aquí nos vamos a centrar en la ciencia debido a que, en la actual crisis, determina en buena medida la acción del Estado y de la sociedad civil. La relación de la ciencia con los poderes públicos y otros sectores sociales, su poder o la falta de él, es un factor fundamental para entender la dinámica de las pandemias. El confinamiento de la población, y la consiguiente paralización de la actividad económica, se debe a precisamente a la influencia que adquiere la ciencia en estos asuntos. 
Esta influencia no es uniforme. Depende en buena medida de la manera en que están organizadas las estructuras de $\mathrm{I}+\mathrm{D}$ en relación con las políticas públicas. La existencia de organismos de interfaz y grupos de asesoramiento científico, dotados de suficientes recursos y legitimidad, y entrenados para transferir el conocimiento disponible a las políticas públicas, influye en la capacidad de acción. Es de esperar que los países donde estas estructuras son más débiles (tengan menos recursos, sean menos independientes o menos ágiles), la influencia de la comunidad científica a la hora de implantar medidas como el confinamiento también sea menor.

La influencia de los científicos también tiene que ver con el margen que dejan las controversias científicas para que intereses políticos o económicos impongan su criterio a favor o en contra de los argumentos de los expertos. Los sociólogos de la ciencia hace mucho que descubrieron que el conocimiento científico de los hechos no es el único factor que interviene a la hora establecer un curso de acción ${ }^{4}$. Las agendas políticas o económicas siempre compiten con el conocimiento disponible debido a los intereses en juego. Ello ocurre sobre todo en problemas científicos de gran complejidad que dan lugar a incertidumbres a la hora de adoptar soluciones que funcionan a largo plazo. En estas circunstancias «se cuelan» constantemente visiones políticas o económicas en conflicto a favor o en contra de una postura. Problemas típicos son: los esfuerzos que se deben hacer para reducir los gases que tienen efecto invernadero, las actuaciones en las construcciones del litoral frente a los riesgos de tsunamis o las inversiones en sanidad para prevenir las pandemias peligrosas a largo plazo, cuando no hay ninguna a la vista.

La actual crisis pandémica ofrece lecciones sobre la relación entre ciencia y política debido al carácter único del fenómeno. Digamos que la ventaja (si es que se puede encontrar alguna en esta crisis) se encuentra en la inmediatez. Lo que ocurre con el COVID-19 es visible en el mundo real y es entendible por prácticamente todo el mundo en un periodo muy corto de tiempo. Por ello se han implantado las medidas de confinamiento. Obviamente hay controversias científicas entre los especialistas sobre el tipo de medidas más efectivas (Elbe et al., 2013). Y las seguirá habiendo. Sin embargo, las controversias entre ciencia y política, o entre ciencia y otros intereses económicos, al decidir el confinamiento se han resuelto en días. Se ha visto una y otra vez cómo muchos políticos se han resistido poniendo por delante supuestos intereses de carácter económico o nacional. Y en muy poco tiempo han tenido que cambiar debido a la fuerza de las evidencias. Ello no quiere decir que los expertos acierten en todo lo referido a las medidas de confinamiento. La ciencia, como cualquier otra institución orientada a la acción, usa los conocimientos disponibles no porque sean necesariamente correctos, sino porque es lo que se tiene (Lamo de Espinosa et al., 1994). Por ahora, el criterio de la ciencia está ganando a base de evidencias que se imponen a corto plazo. La permanencia de criterios similares en fases posteriores de la crisis es un asunto que dependerá del grado de influencia que logren mantener los expertos.

Un cuarto grupo de factores condicionantes tiene que ver con la construcción de instituciones. Algunas de las formas de acción colectiva más poderosas del mundo moderno se encuentran en las instituciones y en sus respectivas organizaciones. La manera en que están configuradas las instituciones en sectores de las políticas públicas y de la economía (los llamados sistemas socio-técnicos) moldea la expansión de la pandemia. Dos sistemas son especialmente importantes en esta crisis: el sanitario y el de I+D. No es este el lugar para hacer un análisis de sus capacidades, aunque una hipótesis importante es que, en condiciones socioeconómicas y culturales similares, el carácter de estos sistemas es determinante en la prevención y en la mitigación de los impactos.

4 Sobre el papel de los científicos en la configuración de las políticas públicas se puede ver Jasanoff (1990). 
Las instituciones son resultado de inversiones en recursos, gestión y reglas formales e informales. En España, donde los contagios son muy superiores a los de otros países europeos debido a la exposición a los viajes y al orden de entrada, la respuesta del sistema de salud depende de las inversiones en recursos materiales y humanos, pero también de la manera en que está construido. Algunos rasgos importantes son el acceso universal, la gestión regida por criterios técnicos y meritocráticos, la rendición de cuentas ante los poderes públicos y los valores de sus trabajadores, más allá de las condiciones de trabajo. La efectividad de este tipo de institución puede ser distinta a la de otros sistemas de salud mucho más ricos, también basados en la profesionalidad y la meritocracia, pero sin acceso universal y con rendición de cuentas dependiente del mercado, como ocurre en los Estados Unidos. La efectividad de un sistema como el español frente a los de otros países europeos, muchos de ellos con un gasto sanitario muy superior al de España, pero con condiciones estructurales distintas respecto a la pandemia, de nuevo es algo que requiere un análisis detallado.

\section{Las consecuencias sociales de la crisis pandémica}

Un asunto central de esta crisis es hasta qué punto una sociedad puede aceptar el riesgo que generarán los impactos sociales del confinamiento y la paralización de la actividad económica más allá de los efectos en la salud. El papel de las ciencias sociales para encontrar criterios de actuación se puede discutir a través de los mismos principios tratados en el punto anterior.

En lo referido a la estructura social es evidente que los impactos se distribuyen desigualmente en la población. Los más inmediatos se aprecian en pocas semanas en los colectivos más vulnerables: inmigrantes irregulares, personas sin hogar, trabajadores precarios y personas que se encuentran en el límite de la subsistencia. A continuación vendrán consecuencias en muchos sectores de actividad. En el terreno de la educación, la enseñanza online tendrá distintos efectos en función del equipamiento de los hogares y las habilidades tecnológicas de las familias. En el terreno del trabajo, en las sociedades del sur de Europa una parte importante de la población depende de la combinación de empleos esporádicos, trabajo informal y solidaridad familiar. Otra parte depende de trabajo independiente y de micro-empresas de menos de cinco trabajadores en el sector servicios. Estos sectores son los más afectados por el confinamiento. La distribución desigual de los costes sociales puede dar lugar a una sociedad mucho más polarizada si no se actúa en consecuencia.

El segundo grupo de impactos puede estar en la cultura y en las formas de vida, que además se retroalimentan con lo anterior. Los efectos de la crisis estarán influenciados por las percepciones del riesgo asociado a los contactos personales. La manera de definir los riesgos puede convertir el distanciamiento en algo permanente si no se atajan los sesgos de percepción. También puede afectar a sectores de la economía. La recuperación de los trabajos en el sector turístico dependerá de cómo se entiendan los viajes, las vacaciones y el ocio en contacto con los demás. Las percepciones pueden ser coherentes con las recomendaciones preventivas de salud, pero también pueden basarse en ideas injustificadas, incluso cuando el riesgo haya desaparecido. Los estudios de percepción social de la ciencia nos indican que la gran mayoría de la población, incluso la más educada, solo puede procesar información científica sobre asuntos complejos a través de «atajos» cognitivos, normalmente a través de la confianza en profesionales e instituciones (Lobera y Torres Albero, 2019). Por ello la gestión de la información científica es importante y puede influir a favor o en contra de las soluciones que se adopten. 
Otro grupo de impactos a gestionar, posiblemente de los más importantes, puede afectar a las políticas públicas y a las instituciones. La actual crisis está siendo un experimento natural para probar cómo funcionan los arreglos institucionales. Es decir, qué instituciones son más efectivas, no solo las dedicadas a la salud, sino también las que se muestran más adecuadas para sortear los impactos sociales y económicos. Aquí el papel de las ciencias sociales puede ser útil para observar las diferencias a través de análisis rigurosos que permitan detectar y monitorizar las intervenciones.

La sociología, además, tiene una especial responsabilidad debido a otro de sus principales axiomas: está equipada conceptualmente para prestar atención a las consecuencias no previstas o no intencionales de la acción. Cuando se toman medidas es necesario prestar atención a los efectos planificados, pero también a otros que pueden surgir debido a que las políticas y las instituciones no actúan en el vacío, sino que están sujetas a expectativas y reacciones sociales que tienen efectos de segundo o de tercer orden. La actual crisis pandémica es también una ocasión para extraer importantes lecciones sobre el funcionamiento de los procesos sociales.

Algunos efectos son simples. Por ejemplo, el anuncio de estado de alarma con días de antelación, sin medidas inmediatas para impedir la movilidad, ha provocado que miles de personas se adelanten a la situación y salgan de viaje, lo que por unos días ha dado lugar a un efecto contrario al que se pretendió. Otras consecuencias no intencionadas son mucho más complejas. La gestión de las consecuencias sociales de esta crisis puede generar condiciones emergentes que terminen provocando resultados inesperados. Para detectarlos se requiere de una planificación basada en la observación detallada. Aunque no es posible predecir acontecimientos concretos, sí puede predecirse razonablemente la dirección más probable de algunas tendencias. Un terreno propicio a que ocurran estas dinámicas es la política de información. Retener información o manipular información sobre el funcionamiento de la pandemia puede tener efectos distintos a los previstos debido a que, si dejan de considerarse como fiables las fuentes oficiales, muchas personas pueden acudir a otras no fiables y terminar actuando en contra de lo que se pretende ${ }^{5}$.

\section{Conclusiones}

La crisis del COVID-19 es sin duda un problema complejo de resolver en términos biológicos y sanitarios, pero será un problema social y económico muchísimo más complejo, al menos hasta que se haya obtenido la vacuna y se eliminen los riesgos hasta la próxima pandemia. Para responder a cuestiones como las anteriores, merece la pena pensar el papel de las ciencias sociales en este tipo de crisis y los desafíos a los que se enfrentan. Algunos desafíos están en la manera de organizarnos. Las observaciones y recogidas de datos que se basan en la interacción personal pueden verse afectadas por un tiempo, lo que obligará a buscar fuentes y metodologías alternativas. La comunicación a través de conferencias, congresos y clases presenciales también requerirá de innovaciones.

Pero posiblemente los retos más importantes se refieren a la orientación del papel de las ciencias sociales para contribuir a resolver los efectos de una crisis como la actual. Unos son de carácter interno a las disciplinas. Se encuentran en la manera en que los profesionales orienten la enseñanza y sus habilidades de investigación y análisis para resolver problemas reales. Otros se encuentran en el terreno de la política científica dirigida a financiar, orientar y utilizar las ciencias sociales. Algunas asuntos importantes son: los criterios que se utilizan para evaluar la investigación que se considera más relevante. La financiación de

5 Un resumen de las evidencias provenientes de la gestión de la información en las epidemias de SARS e Influenza puede verse en UNICEF-IDS (2019). 
las ciencias sociales y su organización en temas, especialidades y equipos efectivos para enfrentarse a problemas sociales. Los tipos de instituciones de interfaz necesarias para poner en contacto el conocimiento de las ciencias sociales con las políticas públicas. Los órganos de asesoramiento que deben implantarse y la manera de elegir a los expertos que interactúan con los gobiernos y las corporaciones. Y finalmente, el papel que deben jugar nuestras universidades y centros de investigación en todo ello. Responder a cualquiera de estos desafíos requerirá de un esfuerzo de organización y planificación mucho más avanzado de lo que hemos conocido hasta ahora en España.

\section{Referencias bibliográficas}

Elbe, Stefan; Leach, Melissa y Scoones, Ian (2013). Pandemic Flu Controversies: What have we learned? Reflections from a Workshop to Discuss Lessons, Policy Implications and Future Challenges. Brighton: STEPS Centre and Centre for Global Health Policy, University of Sussex. En línea: http://steps-centre.org/event/ workshop-pandemic-flu-what-have-we-learned/, consultado el 23 de marzo de 2020.

Jasanoff, Sheila (1990). The Fifth Branch: Science Advisers as Policy Makers. Cambridge, MA: Harvard University Press.

Lamo de Espinosa, Emilio; Torres Albero, Cristóbal y González García, José María (1994). La sociología del conocimiento y de la ciencia. Madrid: Alianza.

Leach, Melissa; Scoones, Ian y Stirling, Andrew (2010): "Governing Epidemics in an Age of Complexity: Narratives, Politics and Pathways to Sustainability”. Global Environmental Change, 20 (3), 369-377.

Lobera, Josep y Torres Albero, Cristóbal (Eds.) (2019). Percepción social de la Ciencia y la Tecnología 2018. Madrid: Fundación Española para la Ciencia y la Tecnología.

Navarro, Vicente (2009). “What we Mean by Social Determinants of Health?”. International Journal of Health Services, 39 (3), 423-441.

Portes, Alejandro (2013). Sociología económica: un análisis sistemático. Madrid: CIS.

UNICEF-Institute of Development Studies (IDS) (2019). Social Science Lessons Learned from Influenza and SARS. Evidence Summary. The Social Science in Humanitarian Action: A Communication for Development Platform. En línea: www.socialscienceinaction.org, consultado el 2 de abril de 2020.

Weitz, Rose (2016). The Sociology of Health, Illness, and Health Care. Londres: Cengage Publishing.

\section{Nota biográfica}

Manuel Fernández Esquinas es científico titular del CSIC. Ha trabajado como sociólogo aplicado y consultor de políticas de innovación para varios gobiernos y organismos internacionales. Investiga sobre la organización de los sistemas de I+D y las estructuras sociales de la innovación. Ha publicado numerosos artículos en revistas como Higher Education, Science and Public Policy y Technological Forecasting and Social Change, entre otras, y en editoriales como Routledge, Edward Elgar y CIS. En la actualidad es presidente de la Federación Española de Sociología y coordinador de la red de investigación ESA «Southern European Societies».

Orcid: http:/ / orcid.org/0000-0001-8677-9462. 